\title{
A Simple Protocol Offering Both Atomic Consistent Read Operations and Sequentially Consistent Read Operations
}

\author{
Michel RAYNAL \\ IRISA / Université de Rennes \\ Campus de Beaulieu \\ 35042 Rennes, France \\ raynaleirisa.fr
}

\author{
Matthieu RoY \\ LAAS-CNRS \\ 7 Avenue du Colonel Roche \\ 31077 Toulouse, France \\ roylaas.fr
}

\author{
Ciprian TUTU \\ Computer Science Dpt \\ Johns Hopkins University \\ Baltimore, USA \\ cipriandjhu.edu
}

\begin{abstract}
A concurrent object is an object that can be concurrently accessed by several processes. Two well known consistency criteria for such objects are atomic consistency (also called linearizability) and sequential consistency. Both criteria require that all the operations on the concurrent objects can be totally ordered in such a way that each read operation obtains the last value written into the corresponding object. They differ in the meaning of the word "last" that refers to physical time for atomic consistency, and to logical time for sequential consistency. This paper investigates the merging of these consistency criteria in a multiprocess program. The proposed combination offers two read operations to the processes, namely, an atomic read operation and a sequentially consistent read operation. While the first provides a process with the last "physical" value of an object, the second provides it with a value that is approximate with respect to real-time but whose semantics is perfectly well defined. A protocol that implements the combination on top of an asynchronous distributed system is described. The protocol provides a better understanding of the similarities and differences between these consistency criteria. Moreover, the protocol is generic in the sense that it can be tailored to provide only one of these consistency criteria.
\end{abstract}

Keywords: Asynchronous Distributed Systems, Atomic Consistency, Combination of consistency criteria, Linearizability, Message Passing, NP-Completeness, Shared Memory Abstraction, Sequential Consistency.

\section{Introduction}

Context of the study The definition of a consistency criterion is crucial for the correctness of a multiprocess pro- gram. Basically, a consistency criterion defines which value has to be returned when a read operation on a shared object is invoked by a process. The strongest (i.e., most constraining) consistency criterion is atomic consistency [12] (also called linearizability [8]). It states that a read returns the value written by the last preceding write, "last" referring to real-time occurrence order (concurrent writes being ordered). Causal consistency $[3,5]$ is a weaker criterion stating that a read does not get an overwritten value. Causal consistency allows concurrent writes; consequently, it is possible that concurrent read operations on the same object get different values (this occurs when those values have been produced by concurrent writes). Other consistency criteria (weaker than causal consistency) have been proposed $[1,21]$.

Sequential consistency [10] is a criterion that lies between atomic consistency and causal consistency. Informally it states that a multiprocess program executes correctly if its results could have been produced by executing that program on a single processor system. This means that an execution is correct if we can totally order its operations in such a way that (1) the order of operations in each process is preserved, and (2) each read gets the last previously written value, "last" referring here to the total order. The difference between atomic consistency and sequential consistency lies in the meaning of the word "last". This word refers to real-time when we consider atomic consistency, while it refers to a logical time notion when we consider sequential consistency (namely the logical time defined by the total order). The main difference between sequential consistency and causal consistency lies in the fact that (as atomic consistency) sequential consistency orders all write operations, while causal consistency does not require to order concurrent writes.

It has been shown that determining whether a given execution is sequentially consistent is an NP-complete problem $[13,23]$. This has an important consequence as it rules out the possibility of designing efficient sequential consistency 
protocols (i.e., protocols that provide sequentially consistent executions and just these). This means that, in order to be able to design efficient sequential consistency protocols, additional constraints have to be imposed on executions. One of these constraints (that has been proposed in [15]) is the following. Let two operations conflict if both are on the same object and one of them is a write. Let us say that an execution satisfies the $O O$-constraint if any pair of conflicting operations are ordered. It is shown in [15] that an $O O$-constrained execution is sequentially consistent if its read operations are legal (i.e., do not provide overwritten values). This approach shows that a sequential consistency protocol can be obtained by combining two mechanisms: one providing the $O O$-constraint and the other providing read legality.

On another side, we have shown in [17] that sequential consistency can be seen as a form of lazy atomic consistency. The main difference between the two lies in the fact that sequential consistency allows a process to keep a cached value as long as it does not make other operations inconsistent, while atomic consistency requires to update or invalidate the cached values of an object as soon as this object is modified.

Content of the paper This paper investigates the combination of sequential consistency with atomic consistency within the same parallel program.

We provide processes with two types of read operations, namely, sequentially consistent read and atomically consistent read. The first type of read can be seen as an approximate read. Differently from previous approaches that allow processes to invoke read operations providing "approximate" values, here all the read operations have a welldefined semantics. A protocol is proposed that implements this type of consistency criteria combination on top of an asynchronous message-passing distributed system. Interestingly, this protocol can be seen as an extension of the sequential consistency protocol presented in [6]. In the full version of the paper we presented a second combination where processes share objects which can be either sequentially consistent or atomically consistent. The interested reader can refer to [19] where the combination is presented together with a protocol that generalizes the sequential consistency protocol of [17].

In addition to its own interest, the proposed protocol provides a better understanding of the link relating sequential consistency and atomic consistency.

Roadmap The paper consists of three sections. Section 2 presents the shared memory abstraction, atomic consistency, and sequential consistency. Section 3 investigates the combination and proposes a corresponding protocol. Finally, Section 4 provides a few concluding remarks.

\section{The Consistent Shared Memory Abstrac- tion}

A parallel program defines a set of processes interacting through a set of concurrent objects. This set of shared objects defines a shared memory abstraction. Each object is defined by a sequential specification and provides processes with operations to manipulate it. When it is running, the parallel program produces a concurrent system [8]. As in such a system an object can be accessed concurrently by several processes, it is necessary to define consistency criteria for concurrent objects.

\subsection{Shared Memory Abstraction, History and Le- gality}

Shared Memory Abstraction A shared memory system is composed of a finite set of sequential processes $p_{1}, \ldots, p_{n}$ that interact via a finite set $X$ of shared objects. Each object $x \in X$ can be accessed by read and write operations. A write into an object defines a new value for the object; a read allows to obtain a value of the object. A write of value $v$ into object $x$ by process $p_{i}$ is denoted $w_{i}(x) v$; similarly a read of $x$ by process $p_{j}$ is denoted $r_{j}(x) v$ where $v$ is the value returned by the read operation; $o p$ will denote either $r$ (read) or $w$ (write). For simplicity, as in [3, 12, 20], we assume all values written into an object $x$ are distinct ${ }^{1}$. Moreover, the parameters of an operation are omitted when they are not important. Each object has an initial value (it is assumed that this value has been assigned by an initial fictitious write operation).

History concept Histories are introduced to model the execution of shared memory parallel programs. The local history (or local computation) $\widehat{h}_{i}$ of $p_{i}$ is the sequence of operations issued by $p_{i}$. If $o p 1$ and $o p 2$ are issued by $p_{i}$ and $o p 1$ is issued first, then we say "op 1 precedes $o p 2$ in $p_{i}$ 's process-order", which is noted $o p 1 \rightarrow_{i}$ op 2 . Let $h_{i}$ denote the set of operations executed by $p_{i}$; the local history $\widehat{h}_{i}$ is the total order $\left(h_{i}, \rightarrow_{i}\right)$.

Definition 1 An execution history (or simply history, or computation) $\widehat{H}$ of a shared memory system is a partial order $\widehat{H}=\left(H, \rightarrow_{H}\right)$ such that:

- $H=\bigcup_{i} h_{i}$

- op $1 \rightarrow_{H}$ op 2 if:

$\vee\left\{\begin{array}{lr}\exists p_{i}: o p 1 \rightarrow_{i} \text { op } 2 & \text { (process-order) } \\ \left(\text { op } 1=w_{i}(x) v\right) \wedge\left(\text { op } 2=r_{j}(x) v\right) & \text { ( read-from ) } \\ \exists o p 3:\left(o p 1 \rightarrow_{H} \text { op } 3\right) \wedge(o p 3 \rightarrow H \text { op } 2)\end{array}\right.$

Two operations op 1 and op 2 are concurrent in $\widehat{H}$ if we have neither op $1 \rightarrow_{H}$ op 2 nor op $2 \rightarrow_{H}$ op 1 .

1 Intuitively, this hypothesis can be seen as an implicit tagging of each value by a pair composed of the identity of the process that issued the write plus a sequence number. 


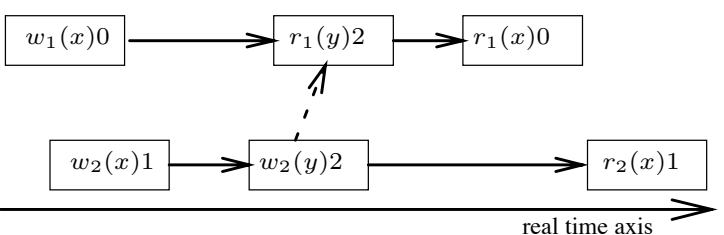

Figure 1. A sequentially consistent execution

Legality notion The legality concept is the key notion on which are based definitions of shared memory consistency criteria $[3,5,7,14,21]$. From an operational point of view, it states that, in a legal history, no read operation can get an overwritten value.

Definition 2 A read operation $r(x) v$ is legal if:

$$
\wedge \begin{cases}\exists w(x) v: & w(x) v \rightarrow_{H} r(x) v \\ \nexists o p(x) u: & (u \neq v) \\ & \wedge\left(w(x) v \rightarrow_{H} \text { op }(x) u \rightarrow_{H} r(x) v\right)\end{cases}
$$

A history $\widehat{H}$ is legal if all its read operations are legal.

\subsection{Sequential Consistency}

Sequential consistency has been proposed by Lamport in 1979 to define a correctness criterion for multiprocessor shared memory systems [10]. A system is sequentially consistent with respect to a multiprocess program, if "the result of any execution is the same as if (1) the operations of all the processors were executed in some sequential order, and (2) the operations of each individual processor appear in this sequence in the order specified by its program".

This informal definition states that the execution of a program is sequentially consistent if it could have been produced by executing this program on a single processor sys$t^{2}{ }^{2}$. More formally, we define sequential consistency in the following way. Let us first recall the definition of linear extension of a partial order. A linear extension $\widehat{S}=\left(S, \rightarrow_{S}\right)$ of a partial order $\widehat{H}=\left(H, \rightarrow_{H}\right)$ is a total order that respects the partial order. This means we have the following: (i) $S=H$, (ii) $o p_{1} \rightarrow_{H} o p_{2} \Rightarrow o p_{1} \rightarrow_{S} o p_{2}(\widehat{S}$ maintains the order of all ordered pairs of $\widehat{H}$ ) and (iii) $\rightarrow_{S}$ defines a total order.

Definition 3 A history $\widehat{H}=\left(H, \rightarrow_{H}\right)$ is sequentially consistent if it has a legal linear extension.

2 In his definition, Lamport assumes that the process-order relation defined by the program (point 2) of the definition) is maintained in the equivalent sequential execution, but not necessarily in the execution itself. As we do not consider programs but only executions, we implicitly assume that the process-order relation displayed by the execution histories are the ones specified by the programs which gave rise to these execution histories.
As an example let us consider the history $\widehat{H}$ depicted in Figure 1 (only the edges that are not due to transitivity are indicated, transitivity edges come from process-order and read-from relations. Moreover, process-order edges are denoted by continuous arrows and read-from edges by dotted arrows). Each process $p_{i}(i=1,2)$ has issued three operations on the shared objects $x$ and $y$. As we can see, when looking at the real time axis, the write operations $w_{1}(x) 0$ and $w_{2}(x) 1$ are concurrent. The $r_{1}(y) 2$ and $w_{2}(y) 2$ are also concurrent. It is easy to see that $\widehat{H}$ is sequentially consistent by building a legal linear extension $\widehat{S}$ including first the operations issued by $p_{2}$ and then the ones issued by $p_{1}$, namely we have:

$$
\widehat{S}=w_{2}(x) 1 \quad w_{2}(y) 2 \quad r_{2}(x) 1 \quad w_{1}(x) 0 \quad r_{1}(y) 2 r_{1}(x) 0 .
$$

This means that $\widehat{H}$ could have been produced by executing the multiprocess program on a machine with a single processor and a scheduler, which is the very essence of sequential consistency.

\subsection{Atomic Consistency}

Atomic consistency is the oldest consistency criterion in the sense that it has always been implicitly used. It has been formalized in [12], and then extended to objects more sophisticated than read/write objects under the name linearizability [8].

Atomic consistency considers that operations take time and consequently its definition is based on the real-time occurrence order of operations. Let $\prec_{r t}$ be a real-time precedence relation on operations defined as follows: $o p_{1} \prec_{r t}$ $o p_{2}$ if $o p_{1}$ was terminated before $o p_{2}$ started ${ }^{3}$. Let us notice that $\prec_{r t}$ is a partial order relation as two operations whose executions overlap in real-time are not ordered.

Definition 4 A history $\widehat{H}=\left(H, \rightarrow_{H}\right)$ is atomically consistent if it has a legal linear extension that includes $\prec_{r t}$.

This means that, to be atomically consistent, $\widehat{H}$ must have a legal linear extension $\widehat{S}=\left(H, \rightarrow_{S}\right)$ such that $\forall o p_{1}, o p_{2}:\left(o p_{1} \prec_{r t} o p_{2}\right) \Rightarrow\left(o p_{1} \rightarrow_{S} o p_{2}\right)$. The linear extension $\widehat{S}$ has to keep real-time order. It is easy to see why invalidation-based atomic consistency protocols use an eager invalidation strategy: this ensures that the real-time occurrence order on operations cannot be ignored, a read always getting the last value (with respect to real-time).

It is easy to see that the execution described in Figure 1 is not atomically consistent (the read operations on $x$ issued by by $p_{1}$ and $p_{2}$ should return the same value, that value being determined by the ordering on $w_{1}(x) 0$ and $w_{2}(x) 1$

3 See [8] for a formal definition of "terminated before" and "started". 


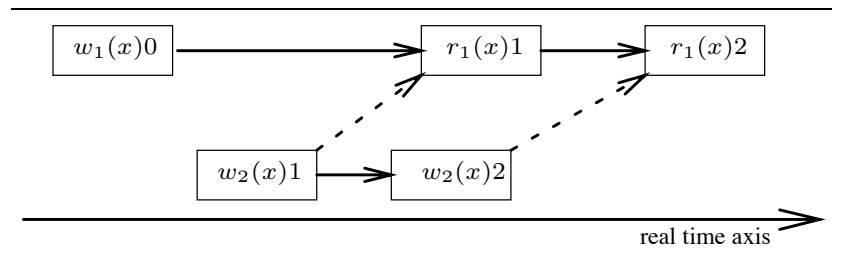

Figure 2. An atomically consistent execution

imposed by the execution). Differently, the execution described in Figure 2 is atomically consistent. A base linear extension $\widehat{S}_{1}$ respecting real time order is the following:

$$
\widehat{S_{1}}=w_{1}(x) 0 \quad w_{2}(x) 1 \quad r_{1}(x) 1 \quad w_{2}(x) 2 \quad r_{1}(x) 2 .
$$

As we can see in $\widehat{S_{1}}$, the concurrent operations $r_{1}(x) 1$ and $w_{2}(x) 2$ have been ordered by the execution with the read operation first. Another execution could have ordered them differently, in that case we would have the base linear extension $S_{2}=w_{1}(x) 0 \quad w_{2}(x) 1 \quad w_{2}(x) 2 \quad r_{1}(x) 2 \quad r_{1}(x) 2$. It is important that to notice that, in all cases, the second read operation by $p_{1}$ obtains the value 2 , as that value is the last value written into $x$ (last with respect to real time).

Atomic consistency vs sequential consistency Atomic consistency and sequential consistency are often confused, even in some textbooks! The fact that one has to respect real time order (atomic consistency) while the other has not (sequential consistency) has a fundamental consequence that has an important impact in practice. It is stated by the following property.

A property $P$ of a concurrent system is local if the system as a whole satisfies $P$ whenever each individual object satisfies $P$. The following theorem is a main result of [8]. It states that atomic consistency is a local property. let $\widehat{H} \mid x(\widehat{H}$ at $x)$ be the projection of $\widehat{H}$ on $x$ (i.e., $\widehat{H} \mid x$ includes only the operations that involve $x$ ).

Theorem 1 [8] $\widehat{H}$ is atomically consistent iff, for each object $x, \widehat{H} \mid x$ is atomically consistent.

This theorem is important as it states that a concurrent system can be designed in a modular way: atomically consistent objects can be implemented independently one from the other. Unfortunately, sequential consistency is not a local property [8]. As we will see in Section ??, the sequentially consistent objects have to cooperate to guarantee sequential consistency. From an operational point of view, this translates as follows. As atomic consistency considers real time while sequential consistency considers logical time, the way these consistency criteria use cached values are very different. Hence, there is a tradeoff between the locality of a consistency criterion and the timeliness (eager vs lazy) of the invalidation strategy used in the protocol that implements it.

\subsection{The Constraint-Based Approach for Sequen- tial Consistency}

As indicated in the Introduction, determining whether a given execution is sequentially consistent is an NPcomplete problem [13, 23]. As we have noticed, this result rules out the possibility of designing efficient protocols providing sequentially consistent histories and just these.

The Constraint-Based Approach Hence, the idea we have developed in [15] that consists in imposing additional constraints on histories in order to be able to design efficient sequential consistency protocols. This approach is similar to imposing constraints on view serializability for concurrency control protocols $[9,16]^{4}$. Let two operations conflict if both are on the same object $x$ and one of them is a write. The two following additional constraints have introduced in [15].

Definition 5 A history $\widehat{H}$ satisfies the $W W$-constraint if any pair of write operations are ordered under $\widehat{H}$.

A history $\widehat{H}$ satisfies the $O O$-constraint if any pair of conflicting operations are ordered under $\widehat{H}$.

Hence, when $\widehat{H}$ satisfies the $W W$-constraint, all its write operations are totally ordered. Differently, when $\widehat{H}$ satisfies the $O O$-constraint, the operations on each object $x \in X$ follow the reader/writer synchronization discipline (for each $x$, the write operations are totally ordered, and the read operations are ordered with respect to the write operations). The following theorems are stated and proved in [15].

Theorem 2 [15] Let $\widehat{H}=\left(H, \rightarrow_{H}\right)$ be a history that satisfies the $W W$-constraint. $\widehat{H}$ is sequentially consistent if and only if it is legal.

Let $\widehat{H}=\left(H, \rightarrow_{H}\right)$ be a history that satisfies the OOconstraint. $\widehat{H}$ is sequentially consistent iff it is legal.

This theorem has important consequences. They mean that, to get sequential consistency, a protocol based on such a constraint only needs to ensure that the read operations are legal. In that way, efficient protocols ensuring sequential consistency can be obtained.

Constraint-Based Protocols for Sequential Consistency Several protocols providing a sequentially consistent shared memory abstraction on top of an asynchronous message passing distributed system have been proposed. Among them, the protocols introduced in $[2,6]$, although they do not explicitly identify the $W W$-constraint, are implicitly based on it. Differently, the protocols presented in $[15,18]$ are explicitly based on the $W W$-constraint.

4 Interestingly, view equivalence can be considered as a special case of sequential consistency, while strict view equivalence can be considered as a special case of atomic consistency. 
The protocol described in [2] implements a sequentially consistent shared memory abstraction on top of a physically shared memory and local caches. It uses an atomic $n$-queue update primitive and so implicitly relies on the the $W W$ constraint. The protocol described in [6] assumes each local memory contains a copy of the whole shared memory abstraction. It orders the write operations using an atomic broadcast facility: all the writes are sent to all processes and are delivered in the same order by each process. Read operations issued by a process are appropriately scheduled to ensure their legality.

The protocol described in [15] considers a server site that has a copy of the whole shared memory abstraction. The local memory of each process contains a copy of a shared memory abstraction, but the state of some of its objects can be "invalid". When a process wants to read an object, it reads its local copy if it is valid. When a process wants to read an object whose state is invalid, or wants to write an object, it sends a request to the server. In that way the server orders all write operations. An invalidation mechanism ensures that the reading by $p_{i}$ of an object that is locally valid is legal. A variant of this protocol is described in [4]. The protocol described in [18] uses a token that orders all write operations and piggybacks updated values. As the protocol described in [6] it provides fast read operations ${ }^{5}$.

A sequential consistency protocol fully based on the $O O$-constraint has been proposed in [17]. This protocol has no centralized control, it is based on a pure distributed control. A sequential consistency protocol for operations that can span several objects is described in [22].

\section{Combination of Atomic Consistency and Sequential Consistency}

\subsection{The Combination}

Considering a set $\Pi$ of $n$ sequential processes $p_{1}, \ldots, p_{n}$ that interact via a finite set $X=\{x, y, \ldots\}$ of shared objects, the first type of combination we propose provides the processes with two types of read operations, namely the operations $a c \operatorname{read}(x)$ and $s c \operatorname{read}(x)$.

- The semantics associated with ac $\operatorname{read}(x)$ is atomic consistency. This means that, when a process invokes ac_read $(x)$, it obtains as a result the last value that has been written into $x$, "last" referring here to real-time order (concurrent write operations appear to the processes as being totally ordered).

5 As shown in [6] atomic consistency does not allow protocols in which all read operations (or all write operations) are fast $[8,14]$. Differently, causal consistency allows protocols where all operations are fast $[3,5$, 20].
- The semantics associated with $s c \operatorname{read}(x)$ is sequential consistency. This means that, when a process $p_{i}$ invokes $s c$ read $(x)$, it obtains as a result the last value of $x$, "last" having here a local meaning: it is the last value of $x$ as known by $p_{i}$ (as long as this value does not compromise the semantics of the other read operations issued by the processes).

\subsection{A Simple Protocol}

This section presents a very simple protocol implementing the previous combination. The underlying system consists of $n$ sites, one per process. The processes communicate through reliable channels by sending and receiving messages. There are assumptions neither on the processing speed of the sites, nor on message transfer delays. Hence, the underlying distributed system is asynchronous. broadcast() is used as a shortcut for forall $p_{j} \in \Pi$ do send () to $p_{j}$ enddo.

We assume that the underlying system provides the processes with the following get_sn () primitive. When invoked by a process, the primitive returns the current value of a shared counter sn (initialized to 0 ). This counter provides sequence numbers as follows: it is increased only when the parameter type of the invocation is equal to $\mathrm{W}\left({ }^{6}\right)$ :

$$
\begin{aligned}
& \text { primitive } \text { get_sn }(\text { type }) \text { : } \\
& \text { if }(\text { type }=\mathrm{W}) \text { then } s n \leftarrow s n+1 \text { endif; } \\
& \text { return }(s n)
\end{aligned}
$$

The protocol is described in Figure 3. Each process $p_{i}$, maintains a copy of each shared object $x$, denoted $C_{i}[x]$. It has also a local counter denoted $l_{-} c t_{i}$ used to count the number of write operations that $p_{i}$ has received so far.

When a process $p_{i}$ invokes a write on an object $x$, it associates the next sequence number to this write invocation (get_sn (W)) and broadcasts it to the whole set of processes (including itself). Then, the write operation completes when $p_{i}$ receives its own write operation.

The implementation of the operation $s c \operatorname{read}(x)$ consists in returning the copy of $x$ that is currently in the local cache, namely $C_{i}[x]$. The implementation of the operation ac $\operatorname{read}(x)$ consists in first obtaining the sequence number of the last write operation (invocation get_sn (R)), then waiting until all the writes up to the last one have been received by $p_{i}$, and finally returning the current value of $C_{i}[x]$.

If we suppress the ac read $(x)$ operation, the protocol becomes the sequential consistency protocol introduced in [6], that is based on an underlying ordered broadcast primitive. So, as noticed in the Introduction, this protocol exhibits a simple connection relating sequential consistency and atomic consistency.

6 There are different ways to implement such a shared counter. One of them is to use a traveling token. 


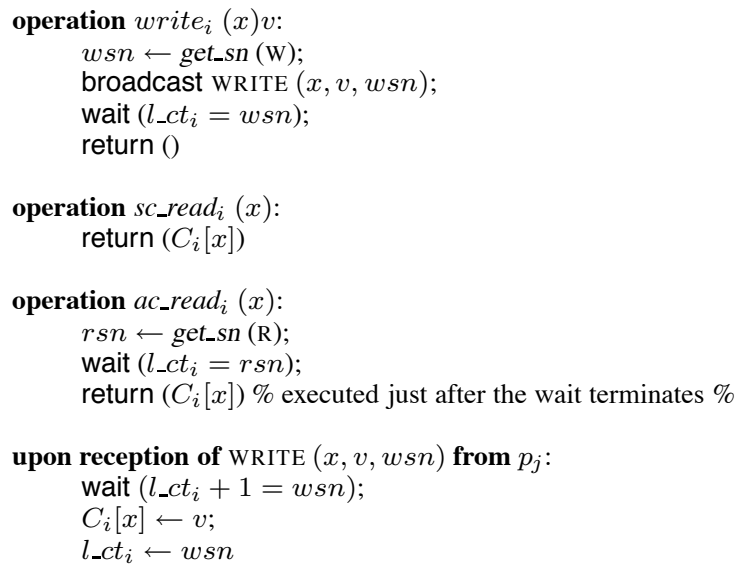

Figure 3. A protocol for combining atomic and sequential consistency

\section{Concluding Remarks}

This paper has explored a possible way to integrate sequential consistency and atomic consistency. Based on a consistent merging of total orders defining these consistency criteria, the semantics of the proposed combination is well defined. The paper has also proposed a protocol implementing this combination. Interestingly, the protocol encompasses previous protocols designed for a single criterion. In that sense the paper provides a better insight on these consistency criteria and the way they can be implemented.

\section{References}

[1] Adve S.V. and Garachorloo K., Shared Memory Models: a Tutorial. IEEE Computer, 29(12):66-77, 1997.

[2] Afek Y., Brown G. and Merritt M., Lazy Caching. ACM TOPLAS, 15(1):182-205, 1993.

[3] Ahamad M., Hutto P.W., Neiger G., Burns J.E. and Kohli P., Causal memory: Definitions, Implementations and Programming. Distributed Computing, 9:37-49, 1995.

[4] Ahamad M. and Kordale R., Scalable Consistency Protocols for Distributed Services. IEEE Transactions on Parallel and Distributed Systems, 10(9):888-903, 1999.

[5] Ahamad M., Raynal M. and Thia-Kime G., An Adaptive Protocol for Implementing Causally Consistent Distributed Services. Proc. 18th IEEE Int. Conf. on Distributed Computing Systems, pp. 86-93, Amsterdam (Netherland), 1998.

[6] Attiya H. and Welch J.L., Sequential Consistency versus Linearizability. ACM Transactions on Computer Systems, 12(2):91-122, 1994.

[7] Garg V.K. and Raynal M., Normality: a Correctness Condition for Concurrent Objects. Parallel Processing Letters, 9(1):123-134, 1999.
[8] Herlihy M.P. and Wing J.L., Linearizability: a Correctness Condition for Concurrent Objects. ACM Transactions on Programming Languages and Systems, 12(3):463-492, 1990.

[9] Ibaraki T., Kameda T. and Minoura T., Serializability with Constraints. ACM Transactions on Database Systems, 12(3):429-452, 1987.

[10] Lamport L., How to Make a Multiprocessor Computer that Correctly Executes Multiprocess Programs. IEEE Transactions on Computers, C28(9):690-691, 1979.

[11] Li K. and Hudak P., Memory Coherence in Shared Virtual Memory Systems. ACM Transactions on Computer Systems, 7(4):321-359, 1989.

[12] Misra J., Axioms for Memory Access in Asynchronous Hardware Systems. ACM Transactions on Programming Languages and Systems, 8(1):142-153, 1986.

[13] Mittal N. and Garg V.K., Consistency Conditions for MultiObject Distributed Operations. Proc. 18th IEEE Int. Conf. on Distributed Computing Systems, pp. 582-589, 1998.

[14] Mizuno M., Nielsen M.L. and Raynal M., An Optimistic Protocol for a Linearizable Distributed Shared Memory System. Parallel Processing Letters, 6(2):265-278, 1996.

[15] Mizuno M., Raynal M. and Zhou J.Z., Sequential Consistency in Distributed Systems. Proc. Int. Workshop on Theory and Practice of Distributed Systems, Springer Verlag LNCS \#938, pp. 224-241, Dagsthul Castle (Germany), 1994.

[16] Papadimitriou C.H., The Theory of Concurrency Control. Computer Science Press, 1986.

[17] Raynal M., Sequential Consistency as Lazy Linearizability. 14th ACM Symposium on Parallel Algorithms and Architectures (SPAA'02), pp. 151-152, Winnipeg (Canada), 2002.

[18] Raynal M., Token-Based Sequential Consistency. Journal of Computer Systems Science and Engineering, 17(6):359-365.

[19] Raynal M., Roy M. and Tutu C., Merging Atomic Consistency and Sequential Consistency. IRISA Research Report \#1629, June 2004, available at www.irisa.fr/bibli/publi/pi/2004/1629/1629.html.

[20] Raynal M. and Schiper A., From Causal Consistency to Sequential Consistency in Shared Memory Systems. Proc. 15th Int. Conf. on Foundations of Software Technology and Theoretical Computer Science (FST\&TCS'95), Springer-Verlag LNCS \#1026, pp. 180-194, Bangalore (India), 1995.

[21] Raynal M. and Schiper A., A Suite of Formal Definitions for Consistency Criteria in Distributed Shared Memories. Proc. 9th Int. IEEE Conference on Parallel and Distributed Computing Systems (PDCS'96), pp. 125-131, 1996.

[22] Raynal M. and Vidyasankar K., A Distributed Implementation of Sequential Consistency with Multi-Object Operations. 24th IEEE Int. Conf. on Distributed Computing Systems (ICDCS'04), pp. 544-551, Tokyo, 2004.

[23] Taylor R.N., Complexity of Analyzing the Synchronization Structure of Concurrent Programs. Acta Informatica, 19:5784,1983 